\title{
Reflets
}

Revue ontaroise d'intervention sociale et communautaire

\section{Les femmes francophones et le travail obligatoire : un enjeu pour l'économie sociale}

\section{Jacinthe Michaud}

Volume 5, numéro 1, printemps 1999

Pratiques et développement économique communautaire

URI : https://id.erudit.org/iderudit/026251ar

DOI : https://doi.org/10.7202/026251ar

Aller au sommaire du numéro

Éditeur(s)

Reflets : Revue ontaroise d'intervention sociale et communautaire

ISSN

1203-4576 (imprimé)

1712-8498 (numérique)

Découvrir la revue

Citer cet article

Michaud, J. (1999). Les femmes francophones et le travail obligatoire : un enjeu pour l'économie sociale. Reflets, 5(1), 93-113. https://doi.org/10.7202/026251ar

Tous droits réservés (C) Reflets : Revue ontaroise d'intervention sociale et communautaire, 1999
Ce document est protégé par la loi sur le droit d'auteur. L'utilisation des services d'Érudit (y compris la reproduction) est assujettie à sa politique d'utilisation que vous pouvez consulter en ligne.

https://apropos.erudit.org/fr/usagers/politique-dutilisation/ 


\title{
Les femmes francophones et le travail obligatoire: un enjeu pour l'économie sociale
}

\author{
Jacinthe Michaud
}

Au cours d'une recherche dans diverses régions de l'Ontario, j'ai interrogé dix-neuf femmes qui touchaient de l'aide sociale pendant l'été 1998. Certaines d'entre elles — peu nombreuses — tentaient de créer leur petite entreprise et étaient sur le point de réussir; d'autres rêvaient d'y parvenir et élaboraient des plans d'action en ce sens. Loin d'être engagées dans la réalisation de tels projets, la plupart des femmes ont su malgré tout développer des stratégies de résistance et des initiatives rendues nécessaires dans leur lutte pour la survie quotidienne. Dans cette quête pour en finir avec le cycle de la pauvreté, elles sont entrées en contact avec certaines ressources du milieu et elles ont établi des liens avec des organismes communautaires et des groupes de femmes qui, en retour, ont validé leurs efforts. C'est ainsi que j'ai pu observer que le discours sur l'économie sociale origine, d'abord et avant tout, du secteur communautaire et résulte de cette rencontre entre les groupes et leur milieu. Ce discours se développe à partir de l'analyse que ces mêmes groupes font des conditions d'existence et des stratégies qu'ils jugent nécessaires. Le paradigme de l'économie sociale est donc composé d'éléments discursifs sur l'augmentation de la pauvreté et de l'exclusion, d'une part et, d'autre part, sur la nécessité de développer des espaces ouverts à la solidarité. Cependant, dans le contexte actuel, l'économie sociale se trouve à côtoyer un autre phénomène, provenant celui-là de l'espace étatique. Il s'agit du travail obligatoire ou «workfare».

L'article qui suit n'a pas pour objectif de prendre partie sur l'économie sociale. Il n'a pas non plus pour objectif d'essayer de 
"Les groupes sont soumis à de multiples influences en vertu de la position qu'ils occupent entre les exigences des institutions administratives et les demandes exprimées par les femmes ellesmêmes.» définir ce qu'il en est. Il s'agit plutôt de proposer quelques pistes de réflexion à propos d'un enjeu qui se dessine en économie sociale, celui de la production discursive à l'intérieur des groupes de femmes à propos d'une catégorie sociale spécifique :les femmes prestataires de l'aide sociale, touchées par le travail obligatoire. Au-delà de la redécouverte d'un secteur d'activités économiques informel, il s'agit de voir que les discours façonnent les profils et caractérisent des comportements particuliers, attribués à des catégories sociales de sujets. L'ensemble des débats au sein des groupes, les outils d'intervention dont ils se servent pour agir contre la pauvreté et l'analyse politique qu'ils proposent à propos des conditions de pauvreté, éclairent leurs prises de décision sur le travail obligatoire. Mais ces débats, ces pratiques, ces analyses ne se produisent pas en vase clos. Les groupes sont soumis à de multiples influences en vertu de la position qu'ils occupent entre les exigences des institutions administratives et les demandes exprimées par les femmes elles-mêmes.

Cette recherche auprès des femmes francophones a obtenu une subvention de l'UniversitéYork. Elle utilise une méthodologie qualitative, proche du récit de vie. Prenant appui sur la nouvelle politique ontarienne "Ontario au travail», les thèmes abordés lors des entrevues sont diversifiés, comme par exemple, la communauté d'origine, les expériences de l'école et de travail, la formation professionnelle, les engagements communautaires, les contacts avec les agents de l'aide sociale, avec les intervenantes et les intervenants d'organismes communautaires et de groupes de femmes, les préjugés sociaux, le racisme ou autres formes de discrimination, le statut de minoritaire francophone et, enfin, les espoirs et les perspectives d'avenir.

Le terme «femmes francophones» utilisé dans cette recherche inclut les femmes dont le français n'est pas toujours la langue maternelle, compte tenu du pays d'origine, mais est devenu leur langue d'usage dans un contexte d'immigration et l'est demeuré, même après plusieurs années. Ceci m'a permis d'entrer en contact avec des femmes de provenances diverses comme l'Afrique, le Maghreb, Haïti, les Caraïbes, en plus de l'Ontario et du Québec. Ces entrevues ont été réalisées principalement dans les villes d'Ottawa, Sudbury, Timmins, Sturgeon Falls et Toronto. 


\section{L'économie sociale en contexte}

«L'économie

solidaire...

s'apparente ici à la

notion de

"développement

économique

communautaire» qui

se développe dans les

interstices laissés

vacants par l'État-

providence..."
L'ensemble du débat autour de l'économie sociale se situe au coeur des grands bouleversements et des transformations qui traversent les domaines du politique et de l'économique. Il révèle la reconstitution des idéologies dominantes et la nature des mutations culturelles en cours. Au coeur de la production discursive se rencontrent divers acteurs, lesquels, suivant l'espace politique qu'il nous est donné de considérer, seront constitués de certains secteurs de l'État, de divers groupes issus du communautaire et de quelques autres identités collectives intéressées à se faire valoir dans la définition du discours et de l'orientation des stratégies.

Dans ce débat, «exclusion» et "économie sociale» sont des concepts abondamment utilisés, en particulier lorsqu'il est question de redéfinir les rapports entre État et société civile (Noël 1996; Lamarche 1998), des modifications au régime de citoyenneté (Roche 1995; McAll 1995; Lesemann et Ulysse 1995; Jenson et Philips 1996; Boismenu et Jenson 1996; Scott 1996; Jenson 1997), de la restructuration de l'économie vers le développement d'activités localisées entre les sphères du politique et de l'économie formelle dans un contexte de mondialisation des marchés (Clarke 1997; Lamoureux 1998). Des mises en garde préviennent l'utilisation sans discernement de ces concepts. Pour certains, l'exclusion est une notion qui ne permet pas l'analyse des conditions d'émergence et du développement des catégories d'exclus (Castel 1995). Le terme d'économie sociale ne fait pas non plus l'unanimité. Au-delà du pour et du contre, ce concept renferme une bonne dose d'imprécision. Car loin d'être nouvelle, l'économie sociale est à distinguer de l'économie solidaire avec laquelle elle est souvent confondue, même si les structures et les formes se renvoient à l'une et à l'autre (Laville 1995). L'économie solidaire, concept mieux connu en France, s'apparente ici à la notion de «développement économique communautaire» qui se développe dans les interstices laissés vacants par l'État-providence, dont le retrait se fait au nom de la responsabilité individuelle et 
"...les formes

nouvelles de ce

nouveau secteur

d'activité ne sauraient

masquer le fait que

l'économie sociale est de beaucoup antérieure à l'État-providence.» de la rationalisation administrative et budgétaire. L'économie sociale est, selon Laville, «un ensemble plus ancien regroupant à la fois les coopératives, les mutuelles et les associations» (1995:163). Mais, comme pour souligner la parenté des structures qui lient l'économie sociale à l'économie solidaire, Laville ajoute que «le projet d'économie solidaire nous apparaît plus comme une résurgence que comme une émergence» (163).

Quoiqu'il en soit, ces termes indiquent des tendances établies depuis un certain temps dans plusieurs espaces d'activités. Tout semble indiquer que des segments de plus en plus larges d'une population d'exclus font l'objet d'une attention particulière, au terme d'un certain mode de prise en charge et de développement dont l'espace se situerait dans l'interstice de l'activité étatique et de l'économie formelle (Lamoureux 1998). C'est dans cet interstice que se retrouvent les groupes communautaires et les groupes de femmes, vecteurs de ce type de développement, d'initiatives économiques et de créativité sociale.

Mais ne s'agit-il véritablement que d'un secteur qui s'établit là où l'État se désengage? Cela reviendrait à dire que ce type d'activités devient possible parce que l'État renonce en partie à ses responsabilités envers le social et à la légitimité qui en découle. Or, nous avons vu avec Laville que les formes nouvelles de ce nouveau secteur d'activité ne sauraient masquer le fait que l'économie sociale est de beaucoup antérieure à l'État-providence. S'agit-il simplement d'un prolongement? À cela il faudrait avouer que les activités des groupes communautaires, des groupes de femmes, les regroupements associatifs et de bénévoles ne joueraient qu'un rôle de courroie de transmission dans les efforts de l'État pour continuer à investir dans le social. Il faudrait pour cela traiter ce secteur comme un ensemble fixe et uniforme et faire fi des divergences, au sein des groupes de femmes, lorsque vient le temps de débattre de l'autonomie des organisations et éviter la récupération des enjeux. S'agit-il d'une interface (Lamoureux, 1994)? Le concept ici renvoie à une réalité fort complexe des relations entre l'État et les groupes communautaires et à la nature de ces relations. Ainsi, les frontières deviennent le lieu d'un rapport de force. Le champ qui se dessine à l'intersection de l'État, du 
marché et de l'économie sociale devient donc le terrain sur lequel des acteurs collectifs, de forces inégales, mais dont la légitimité a été préalablement reconnue (Jenson 1986; 1988) interviennent pour défendre leurs intérêts, déterminent une nouvelle façon de définir la pauvreté et les besoins des couches sociales touchées.

Caractériser ce qui se dégage de cette nouvelle réalité n'est pas si simple. Même si on assiste, dans l'ensemble des sociétés industrialisées en particulier, à une restructuration et une mondialisation sous la gouverne d'un néolibéralisme pur, même si les discours dominants prônent la responsabilité des citoyens et des communautés (Arendt 1997; Jenson 1997), même si nous assistons à une réelle dévolution vers le local et à un désengagement des services publics, l'activité et l'intervention étatiques, selon les États, et ici selon les provinces, exige que chaque cas étudié soit replacé à l'intérieur de son contexte particulier. Mais il semble que ce qui se joue sur les frontières de l'État et de la société civile - entre groupes de femmes et certaines catégories sociales de femmes vivant de pauvreté, entre économie formelle et économie informelle - devient tout aussi important que ce qui se joue à l'intérieur même de chacune de ces zones prises séparément.

Les interstices de l'économie formelle et de son mode de production dominant à l'intérieur des économies occidentales ont toujours renfermé des zones grises, plus ou moins visibles, au chapitre des activités qui génèrent de la croissance. À l'intérieur

"...on a souvent fait état, et même dénoncé, cette invisibilité de l'ensemble des activités des femmes..." des théories et des analyses féministes par exemple, on a souvent fait état, et même dénoncé, cette invisibilité de l'ensemble des activités des femmes, non seulement tout le travail réalisé à l'intérieur de l'espace social, mais aussi celui tout aussi réel qui se déroule à l'intérieur de l'espace économique même (Waring 1988). D'autant plus que les discours actuels sur l'économie sociale, règle générale, ne parviennent que partiellement à intégrer les principes féministes ou une analyse des rapports sociaux de genre (Côté 1995 :111). La raison de cette difficulté se trouve sans doute du côté des enjeux sur lesquels les groupes de femmes se sont penchés, comme le corps, la violence et l'autonomie, des enjeux que la gauche a du mal à intégrer à l'intérieur des problématiques qui impliquent le pouvoir, l'économie, les luttes sociales. 


\section{L'activité des groupes de femmes dans l'économie sociale}

«Les actions contre la pauvreté, l'aide à la réinsertion sur le marché du travail, les initiatives de développement régional, ont composé depuis longtemps des segments importants du mouvement des femmes.»
La mise sur pied de projets d'entraide tels que les cuisines communautaires ou autres modèles de solidarité pour venir en aide à des familles à bas revenus peut sembler emblématique, mais ceux-ci ne représentent qu'une forme d'activités qualifiées d'économie sociale. Nous pourrions répertorier presque autant de types ou de modèles de ces activités qu'il existe de regroupements. Les actions contre la pauvreté, l'aide à la réinsertion sur le marché du travail, les initiatives de développement régional, ont composé depuis longtemps des segments importants du mouvement des femmes. Bien sûr, l'attention a porté sur des dossiers brûlants, tels la violence, le contrôle du corps, l'égalité juridique, la discrimination au travail, pour ne nommer que quelques exemples. Mais une partie significative des énergies féministes, même si exercées dans l'ombre parfois, a été consacrée à l'amélioration des conditions économiques des femmes. De sorte que, des moments forts tels que les marches des femmes contre la pauvreté «Du pain et des roses» organisées au Québec d'abord, puis à l'échelle nationale, et bientôt au niveau international, ont pu être possibles en raison des capacités de mobilisation déjà présentes dans un très grand nombre de milieux.

Ce qu'il faut comprendre, c'est que la plupart des groupes de femmes se donnent pour mandat de transformer les conditions de vie des femmes, de faire en sorte qu'elles parviennent à l'autonomie, à la prise en charge, au développement des réseaux d'échanges et de solidarité (Femmes en tête 1990). Certains rapports de recherche et de mise à jour de répertoires sur les activités des groupes mentionnent la question du travail tout autant que la violence, comme secteur d'intervention prioritaire pour accomplir ce mandat (Anadón et al. 1992; CSF 1996). Avec des répertoires d'activités aussi diversifiées, il est difficile d'en arriver à certaines définitions comme par exemple, ce qu'on entend par développement régional ou de se demander comment une maison 
"...les groupes de femmes ont de plus en plus adapté leur discours et leurs pratiques en fonction des nouvelles activités économiques qu'ils génèrent...» d'hébergement participe au développement économique communautaire au même titre qu'un groupe sur la réintégration des femmes sur le marché du travail. Disons simplement ceci: les groupes de femmes sont créateurs d'emplois directs et indirects (CSF 1996). Ainsi, une équipe de recherche sur le Saguenay Lac Saint-Jean a pu trouver que les groupes étaient responsables de 90 emplois dans cette région (Anadón, Masson, Tremblay et Tremblay 1992:1). Sans doute est-ce pour cette raison que les groupes de femmes ont de plus en plus adapté leur discours et leurs pratiques en fonction des nouvelles activités économiques qu'ils génèrent (Côté 1995) et misent sur une nouvelle légitimation du communautaire dans le contexte du partenariat avec d'autres acteurs sociaux (Lamoureux 1994 :46).

Les groupes travaillent étroitement avec les femmes mais cela ne veut pas dire nécessairement qu'ils ont la même production discursive ni les mêmes orientations d'action. Par exemple, la décision de s'engager dans le programme «Ontario au travail» en acceptant des placements de travail obligatoire dépendra de multiples facteurs à l'intérieur d'un groupe. D'une part, la dynamique de la production discursive à l'intérieur des groupes se développe et évolue selon l'analyse féministe qui se fait à propos des conditions d'existence, des besoins du milieu, de l'intégration de la diversité des femmes à l'intérieur des structures organisationnelles, du contexte politique, économique et social permettant l'élaboration d'un plan d'action. De ce point de vue, on considérera la production discursive, non seulement selon les ressources disponibles à une organisation mais aussi selon la rencontre entre, d'une part, ce que les femmes disent d'elles-mêmes et d'autre part, ce que les groupes comprennent de cette autoreprésentation.

De plus, cette production discursive est façonnée sur un autre plan, celui des institutions administratives et autres acteurs sociaux, politiques et économiques, intéressés au débat sur la configuration des politiques sociales. C'est le rapport avec certains paliers administratifs de l'État, aujourd'hui déclaré néo-libéral, lequel se doit d'être replacé dans son contexte, c'est-à-dire selon qu'il valide ou invalide les activités du secteur communautaire en regard de l'économie sociale. 
"C'est à l'intérieur du présent contexte de reconnaissance officielle de l'économie sociale que les groupes de femmes devront poursuivre le débat sur la participation/nonparticipation, sur le sens à apporter au partenariat et aux pièges de la complémentarité...»
Car l'économie sociale existe avec ou sans la reconnaissance de l'État. Néanmoins, même si ce secteur évolue à l'intersection de l'État, rendu visible dans le contexte du désengagement de ce dernier et du marché, dont les transformations récentes invitent à de nouvelles formes de solidarité, il y aurait lieu de s'interroger sur la relative autonomie de l'espace réservé à l'économie sociale. Au Québec, le gouvernement du jour a choisi la voie de la reconnaissance et de la collaboration entre partenaires sociaux et économiques. Cette reconnaissance suit la logique établie par l'évolution des rapports entre l'État et les groupes depuis le milieu des années 80 . C'était l'époque où le gouvernement d'alors s'était engagé dans la reconnaissance des groupes de femmes comme partenaires afin d'établir le plan d'action de politiques et de réformes qu'il entendait réaliser. C'est à l'intérieur du présent contexte de reconnaissance officielle de l'économie sociale que les groupes de femmes devront poursuivre le débat sur la participation/non-participation, sur le sens à apporter au partenariat et aux pièges de la complémentarité (Lamoureux 1994; Michaud 1997). En Ontario, la situation se présente tout à fait différemment. Non seulement n'y a-t-il pas de reconnaissance institutionnelle de l'économie sociale, il n'y a pas non plus de reconnaissance de l'action des groupes communautaires et de femmes au sens de l'analyse et de l'expertise du milieu où ils s'insèrent. Pourtant, la réforme de l'aide sociale, pour ne mentionner qu'elle, entend faire porter le volet "placement», inhérent au travail obligatoire, sur le dos des groupes communautaires et de femmes, et ce, à l'intérieur d'une production discursive étatique qui met l'accent sur la responsabilité du citoyen et de la communauté (Arendt 1997; Cholette 1998).

Il existe peu d'analyses comparatives entre l'Ontario et le Québec dans le domaine qui nous intéresse, c'est-à-dire la production du discours des femmes vivant de l'aide sociale et de celui des groupes qui travaillent sur des enjeux tels que la pauvreté, l'économie sociale ou le travail obligatoire (Deniger et al. 1995; Jenson 1997;Andrew 1998). Mais en Ontario comme au Québec, les groupes de femmes et les organismes communautaires élaborent des plans d'intervention dans la communauté sur des questions 
spécifiques comme la pauvreté, la santé, la violence physique et sexuelle, l'éducation et autres questions sociales. Ils travaillent étroitement avec les femmes et témoignent de la détérioration sociale et économique qui les affecte. Plusieurs de ces organisations ont parmi leurs membres des femmes qui vivent de l'aide sociale et qui sont visées par le travail obligatoire dont les applications tendent à se développer partout dans les pays occidentaux (Dandurand 1996).

Bref, dans la cause qui nous intéresse, les groupes de femmes adopteront une stratégie vis-à-vis le travail obligatoire dans leurs propres organismes en fonction de plusieurs évaluations. En admettant que ces évaluations du contexte qui les préoccupe soient diversifiées et complexes, les stratégies qui s'en dégagent tendent à répondre à un dilemme pouvant se résumer ainsi: refuser de participer à l'oppression économique des femmes ou prendre en considération le fait qu'elles risquent d'encourir des sanctions monétaires qui menacent leur survie; devenir un employeur comme les autres qui policent les prestataires d'aide sociale ou donner aux femmes la chance d'échapper à la contrainte d'un placement dans un milieu de travail hostile; développer et multiplier les activités de réseautage en fonction de la solidarité entre mouvements sociaux et parties d'un même mouvement ou participer au programme pour ne pas s'attirer les foudres des organismes subventionneurs (Table féministe de concertation provinciale 1998).

\section{L'auto-représentation des femmes comme production discursive}

S'il y a nécessité de mettre en contexte la production discursive des groupes, il s'avère important de contextualiser également cette diversité de productions discursives avec ce que les femmes disent d'elles-mêmes et de leurs besoins. Il faut garder en tête que les groupes ne rejoignent pas toutes les femmes francophones 
"C'est donc dire que les femmes prestataires sont capables de se dire elles-mêmes, de s'auto-représenter dans les lieux, les espaces publics, les groupes où elles agissent de manière autonome.» prestataires de l'aide sociale et une large proportion d'entre elles demeure en dehors du champ communautaire. Cependant, selon la représentation identitaire des femmes et aussi selon le degré de réceptivité des groupes à leur réalité, elles sont aussi en position de modifier la manière avec laquelle les groupes, à leur tour, les représentent à l'intérieur des espaces publics où ils interviennent. C'est donc dire que les femmes prestataires sont capables de se dire elles-mêmes, de s'auto-représenter dans les lieux, les espaces publics, les groupes où elles agissent de manière autonome. Cette auto-représentation ne peut pas être assimilée au discours produit par les groupes de femmes. Ce n'est pas parce que ces groupes représentent et défendent les intérêts des prestataires que la voix de celles-ci doit être confondue dans le tout indifférencié de leur plate-forme politique.

Il est vrai que les femmes et les collectivités dans lesquelles elles évoluent sont constituées par un ensemble de discours, d'institutions, de pratiques et de procédures et qu'il devient alors possible d'intervenir sur elles en tant qu'objets. Mais les femmes sont aussi des sujets, pouvant éventuellement se gérer elles-mêmes. Colette St-Hilaire illustre bien ce phénomène lorsqu'elle parle des programmes de développement et de leur impact sur les femmes aux Philippines. Elle démontre qu'autour des programmes de développement se constitue tout un dispositif de savoir/pouvoir propre à produire un sujet-femme adapté aux programmes de développement. Elle ajoute que «le savoir, y compris les savoirs féministes, dessine peut-être les figures d'une nouvelle forme de gestion sociale des femmes, contribuant à leur assujettissement» (1996:85).

Mais pour aussi contraignants et réducteurs que puissent être ces dispositifs de production, les femmes adoptent des stratégies d'action qui démontrent qu'il existe des failles et des brèches grâce auxquelles elles entendent résister. C'est donc dire que l'autoreprésentation des femmes francophones doit pouvoir être mise en contraste et parfois même en opposition avec d'autres productions discursives, produites par des formations sociales très variées.

La prise en considération de cette diversité est essentielle, tout simplement parce que les discours sur les assistés sociaux et les assistées sociales en Ontario proviennent de sources multiples; 
tous et chacun concourent à définir les termes et les besoins des prestataires, soit en fonction d'une réintégration au marché du travail, soit en fonction d'une analyse des conditions de pauvreté et de l'oppression spécifique des femmes.

Les femmes vivant de l'aide sociale, par exemple, ont des contacts réguliers avec des représentants et représentantes de diverses institutions et organismes, tels l'aide sociale, les administrations scolaires et de santé, des organismes communautaires de quartiers et des groupes de femmes. Elles peuvent témoigner de la perception que ces personnes, avec qui elles établissent des relations plus ou moins étroites, manifestent par rapport à leur statut: celui de prestataire d'aide sociale mais aussi celui de mère seule avec un ou plusieurs enfants, de noire, francophone, vivant avec un handicap, de jeune ou de personne âgée avec une orientation sexuelle soidisant marginale. Voici le témoignage d'une femme à propos des préjugés qu'elle a dû affronter lors d'un débat sur le travail obligatoire au sein d'un organisme de sa communauté.

Mais il y avait certains entrepreneurs qui voyaient le workfare comme une bonne chose. T'es là, t'es autour de la table et t'écoutes tout ça, pis làj'ai dit «non». Ce monsieur-là m'aime ben gros. Ce monsieur-là, il sait comment est-ce que je suis dévouée. Je vais enfin lui admettre qui je suis. "Tu sais le monde que tu étiquettes depuis tout à l'heure, tu étiquettes moi ici». Il me

"Je lui dis: "Je te l'ai jamais admis, mais moi ça fait cinq ans que je suis sur les prestations familiales». Il s'est fermé tout raide.» regarde. "Qu'est-ce que tu veux dire par ça?" Je lui dis: "Je te l'ai jamais admis, mais moi ça fait cinq ans que je suis sur les prestations familiales». Il s'est fermé tout raide. "Tu sais, il $y$ en a peut-être un pourcentage qui sont sur les prestations que peut-être que c'est comme ça qu'ils ont été élevés, pis que pour eux c'est un mode de vie. Mais les trois quarts des gens, là, sont pris dans ce cercle vicieux-là, pour d'autres raisons, soit une séparation, soit euh... des coupures d'emploi. T'essaies d'avoir un emploi, t'es sur l'assurance chômage. Mais quand ton assurance chômage est terminée, où est-ce que tu vas si tu as une famille à faire vivre?». C'est là que j'ai commencé à lui faire ouvrir les yeux, 
"...les femmes

francophones qui vivent de l'aide sociale ne sont pas si fragmentées que cela dans leur autoreprésentation.» en disant wow là. "L'étiquette que tu mets sur les gens, penses-y ben comme il faut avant d'y mettre». C'était la première fois que j'admettais au monde autour de la table que j'étais sur les prestations.

Il n'est pas facile de catégoriser les femmes francophones, prestataires de l'aide sociale, en tant qu'entités homogènes.Actuellement forcées au travail obligatoire, elles different entre elles à propos de leur expérience de l'école, de la famille, du travail, de la communauté. Mais en dépit de cette diversité des expériences et de leur position divergeante sur le travail obligatoire, les femmes francophones qui vivent de l'aide sociale ne sont pas si fragmentées que cela dans leur auto-représentation. Qu'elles soient dans la jeune vingtaine ou dans la cinquantaine avancée, qu'elles soient d'origine africaine, haïtienne, québécoise ou franco-ontarienne, elles vivent de pauvreté, doivent faire face aux multiples préjugés, subissent les pressions d'un système qui met leur sécurité en danger, augmente leur pauvreté et leur vulnérabilité. En dépit des préjugés et des mythes qui circulent à propos des assistés sociaux en général et des femmes en particulier (Whitmore et Kerans 1988), la désorganisation et l'impuissance à redresser leur vie sont des qualificatifs qui leur sont attribués plus souvent qu'autrement mais qui restent à démontrer. Le témoignage qui suit est un bon exemple de la lutte constante que les femmes doivent mener pour s'en sortir.

Mais j'ai dit: "Je veux me battre, je travaillerai un jour». C'est ça, ce qui me tient, parce que je suis quelqu'un qui, quand je me décide, je dis: "Je vais arriver au bout [...]” Les gens se demandent un peu, comment je fais avec quatre enfants, je vais à l'école, je suis toute seule avec mes quatre enfants.

Et plus loin elle ajoutera :

J'ai fait des examens avec mon enfant sur mon épaule, l'examen de comptabilité. [....] il dormait, j'ai fait l'examen comme ça. Jusqu'à la fin j'ai tenu bon. J'ai terminé la session au mois d'avril. 
Outre les maigres prestations, les femmes ne reçoivent aucune aide du système. Elles vivent de petits boulots, s'inscrivent à des programmes de formation et d'études, s'organisent pour obtenir des pensions alimentaires, luttent contre des décisions arbitraires du système. Parler, dénoncer, vouloir agir sur leur vie et celle de leurs enfants sont des manifestations de résistance qui doivent être mises au compte de leur auto-représentation.

Ce discours est partiellement validé par les groupes de femmes qui appuient et supportent cette résistance, bien que leur analyse et leurs orientations politiques puissent différer d'un regroupement à un autre. Je dis partiellement parce que les groupes doivent faire la part de positions souvent inconciliables. Par exemple, parmi les prestataires d'aide sociale, il y a celles qui appuient le travail obligatoire et celles qui le condamnent absolument.

\section{Discussion : le dilemme des groupes de femmes vis- à-vis la réforme ontarienne}

Les groupes de femmes s'investissent de la responsabilité de représenter les besoins spécifiques des femmes, quelle que soit l'orientation politique choisie. Cependant, il faut relativiser le poids de chacun des acteurs dans le contexte actuel de la politique. Le programme «Ontario au travail» ne fait pas appel à la concertation des groupes mais s'appuie sur le communautaire pour sa réalisation (Cholette 1998). Le fait que les groupes n'aient pas été investis de légitimité d'acteur dans l'élaboration de la politique ne les dispense nullement de se questionner sur l'enjeu de la participation/non-participation, dans le contexte d'une plus grande visibilité de l'économie sociale et/ou du développement du travail obligatoire.

Faisons ici un petit détour. Il n'est pas tout à fait certain que le vieux débat sur la participation/nonparticipation aux réformes et aux programmes de l'État soit devenu dépassé au sein des groupes. Les positions réciproques sur l'économie sociale ne 
«...ces opposés présentent un tableau binaire qui ne reflète pas la complexité et la diversité des positions." seraient en fait qu'une manière de reproduire l'affrontement des tendances sur la participation/non-participation sous une autre forme. Résumons simplement. Au début des années 80, les positions des groupes de femmes pouvaient épouser les deux tendances suivantes (Michaud 1992). D’un côté, il y a la justification de la participation sur la base de l'incontournable réalité de l'État (Regroupement provincial des maisons d'hébergement et de transition 1989; Franzway et al. 1989) et de l'effet de ses politiques dans la vie des femmes. La participation devient un moindre mal, c'est-à-dire que les dangers sont réels entre partenaires inégaux, mais comme les absents ont toujours tort, la nécessité de contrer des discours néfastes obtient la priorité dans les stratégies d'action. À l'opposé, le refus de la participation est basé sur la rareté des énergies, la crainte de la récupération sans reconnaissance de l'expertise et de l'analyse féministes et l'impossibilité de faire valoir l'idéologie du mouvement des femmes au sein de l'État-providence sans diluer son contenu (Relais-femmes 1985).

Sans doute, ces opposés présentent un tableau binaire qui ne reflète pas la complexité et la diversité des positions. D’une part, à peu près tous les groupes connaissent les pièges de la participation trop étroite avec l'administration gouvernementale et savent qu'ils négocient à l'intérieur d'un rapport de force inégal. Et de l'autre, même en supposant qu'il soit possible de refuser la participation et la concertation, la nature des services au sein des groupes exige qu'ils établissent des liens avec certains secteurs du réseau institutionnel (Michaud 1996).

Curieusement, on peut se demander si le débat sur le bienfondé de l'économie sociale s'apparente à celui qui vient d'être exposé plus haut. D'un côté, on soutient que l'économie sociale pourrait créer des emplois pour lesquels les femmes seraient les principales bénéficiaires. Et de l'autre, on avance l'argument que l'économie sociale pourrait représenter un réservoir de maind'oeuvre à bon marché, voire même de travail obligatoire (Boivin 1998). L'affrontement sur ce sujet se situe au niveau des gains que les femmes pourront tirer de l'économie sociale: seront-elles capables de se donner une expérience propre à leur réinsertion 
"Ce débat au sein des groupes communautaires et des groupes de femmes à propos de l'économie sociale doit pouvoir tenir compte de deux choses: le rapport à l'État, quelle qu'en soit la nature, et la façon dont les groupes entendent représenter les femmes à l'intérieur de ce rapport.» sur le marché du travail ou resteront-elles enfermées dans un secteur qui n'a rien d'autre à leur offrir qu'un statut de travailleuses précaires? Plutôt que de gérer la richesse, l'économie sociale ne serait-elle pas plutôt une façon de gérer la pauvreté? Ou est-ce véritablement un secteur porteur d'avenir propre à concurrencer l'économie formelle et le travail salarié qui montrerait des signes de déclin?

Ce débat au sein des groupes communautaires et des groupes de femmes à propos de l'économie sociale doit pouvoir tenir compte de deux choses: le rapport à l'État, quelle qu'en soit la nature, et la façon dont les groupes entendent représenter les femmes à l'intérieur de ce rapport. Premièrement, la volonté de faire valoir la légitimité du développement du secteur de l'économie sociale et le désir d'en rendre visibles les retombées doivent tenir compte des efforts de l'État pour imposer le travail obligatoire à tout prix. Dans ce contexte, la création d'emplois à l'intérieur de l'économie sociale risque d'être tributaire du nombre de placements des prestataires d'aide sociale dans le même secteur, peu importe qu'un ou plusieurs groupes aient pris position contre ce type de placements. Les groupes doivent faire davantage que refuser le travail obligatoire. Ils doivent continuer à se questionner sur les tendances de l'économie sociale à l'intérieur du contexte de la restructuration de l'économie et de l'État.

En deuxième lieu, les groupes s'investissent d'une certaine légitimité, voire d'autorité, lorsqu'il s'agit de représenter la voix des femmes. Or, la représentation des femmes n'est pas si simple. Compte tenu de la diversité des situations des femmes, cette représentation ne peut certainement pas être unitaire et la production discursive doit pouvoir intégrer les facteurs de discrimination tels que la race, la langue, le statut de prestataire. Ce qui se dégage des entrevues évoquées au tout début et dont l'analyse définitive reste à compléter, c'est que les femmes, globalement, ne veulent pas toujours séparer les moments où elles peuvent identifier clairement le facteur déterminant qui leur nie entre autres le droit au logement ou à un emploi décent. Même si toutes vivent de la discrimination par le simple fait d'être assistées sociales, pour plusieurs d'entre elles, le fait d'être une francophone 
de couleur est un élément déterminant dans l'établissement de ce statut. Certes, les groupes de femmes ont fait l'apprentissage, parfois pénible, de la diversité. Ce n'est plus tant le désir d'inclusivité qui risque de faire défaut - même si le perfectionnement du discours à ce chapitre reste à faire - mais d'intégrer toute la complexité d'un problème au coeur de l'analyse. D'autant plus qu'aux facteurs de race, de langue et du statut de prestataire, s'ajoute la réalité de celles qui sont seules avec un ou plusieurs enfants d'âge variable, vivent avec un handicap, ont des niveaux d'éducation et d'expertise qui ne leur sont pas reconnus. La liste de l'ensemble des éléments risque d'être longue, mais chacun d'eux est déterminant dans la vie des femmes. Dès lors, le piège qui attend bien des groupes, c'est de pallier au plus pressé : accepter des placements de travail obligatoire parce que les femmes le demandent.

\section{Conclusion}

Les groupes de femmes, lorsqu'ils débattent du travail obligatoire, arrivent à prendre position en fonction de plusieurs facteurs mais aussi en fonction de plusieurs niveaux d'influence. D'une part, les discours et les pratiques des groupes de femmes sont élaborés à partir de leurs perceptions des besoins des femmes. Plusieurs de ces groupes ont commencé à donner un sens à leur mandat visavis ces besoins spécifiques en des termes qui les rapprochent du paradigme du développement. Ce déplacement de sens signifie que les groupes prennent au sérieux leur capacité d'améliorer la situation économique des femmes, en particulier les plus vulnérables. Au coeur de ce débat interne, l'auto-représentation des femmes émerge et influence la production discursive des groupes de femmes. D'autre part, les pressions exercées sur les groupes pour qu'ils acceptent des placements de travail obligatoire peuvent s'avérer très fortes, non pas seulement du côté des femmes elles-mêmes, mais aussi du côté des instances administratives responsables de l'implantation du programme «Ontario au travail». Les débats sur les orientations politiques au sein des groupes ne se déroulent donc jamais en vases clos. Les groupes de femmes 
émergent et agissent à l'intérieur, comme à l'intersection de plusieurs ensembles. Tout en tenant compte de ces divers niveaux d'influence, force est de constater que c'est dans les relations entre les groupes et les femmes que se joue un rapport de force tout à fait particulier et déterminant. Les groupes doivent vivre pleinement ce rapport avec tout ce qu'il comporte d'affrontements et de déchirements. C'est le prix à payer pour adopter une position qui tienne et dont les femmes ne feront pas les frais à long terme.

\section{Bibliographie}

ANADÓN, Marta, Dominique MASSON, Marielle TREMBLAY et Pierre-André TREMBLAY (1992). De l'organisation aux pratiques d'autodéveloppement : portrait de groupes de femmes du SaguenayLac-Saint-Jean, Équipe «Femmes et développement régional», Chicoutimi Université du Québec à Chicoutimi, $97 \mathrm{p}$.

ANDREW, Caroline (1998). «Les femmes et l'État-providence : question revue et corrigée», Politique et Sociétés, vol.17, nos 1-2,171-182.

AREND, Sylvie (1997). L'idéologie néolibérale et la dépendance des femmes, Forum de la Table féministe francophone de concertation provinciale et du Centre de Recherche féministe de l'Université York, 22 mars.

BOISMENU, Gérard et Jane JENSON (1996). «La réforme de la sécurité du revenu pour les sansemploi et la dislocation du régime de citoyenneté canadien», Politique et société, no 30, 2952.

BOIVIN, Louise (1998). «L'économie sociale : un gisement de travail obligatoire», Louise Boivin et Mark Fortier (s. la dir. de) L'économie sociale : L'avenir d'une illusion, Montréal, Fides, 161-185.

CASTEL, Robert (1995). «Les pièges de l'exclusion», Lien social et politiques -RIAC, vol. 34, automne, 13-21.

CHOLETTE, Chantal (1998). «Le travail obligatoire en Ontario : solution ou imposture?», Reflets, vol. 4 , no 1 .

CLARKE, Tony (1997). «The Transnational Corporate Agenda Behind the Harris Regime», Diana Ralph, André Régimbald et Nérée St-Amand (ed), Mike Harris's Ontario: Open for Business, Closed to People, Halifax Fernwood Publishing, 28-36.

CONSEIL DU STATUT DE LA FEMME (1996). Partenariat État/communautaire: Les groupes de femmes y gagnent-ils au change?, Maude Rochette (rédaction), Québec, Conseil du Statut de la femme, $41 \mathrm{p}$.

CÔTÉ, Denyse (1995). «Développement communautaire et rapports sociaux de sexes : essai d'analyse sur toile de fond régionale», Denise Côté et al. (dir) Du local au planétaire : réflexions et pratiques de femmes en développement régional, Montréal, Remue-ménage, 109-130.

DANDURAND, Pierre (1996). «La question sociale : Réflexions en marge d'un ouvrage de Pierre Rosanvallon", Sociologie et sociétés, vol. 28, no 2, 189-198.

DENIGER, Marc-André, R. Scott EVANS, Viviane PORTEBOIS, Monique PROVOST, André 
RINGIMBALD et Jean-François RENÉ (1995). Pauvreté et insertion sociale et professionnelle de jeunes familles: une comparaison Québec/Ontario, Ottawa, Conseil de planification sociale d'OttawaCarleton/Conseil canadien de développement social, $141 \mathrm{p}$.

FEMMES ENTÊTE (1990). De travail et d'espoir: des groupes de femmes racontent le féminisme, Montréal, Remue-ménage.

FRANZWAY, Suzanne, Dianne COURT et R.W. CONNELL (1989). Staking a Claim : Feminism, Bureaucracy and the State, Cambridge, Polity Press.

JENSON, Jane (1997). «Fated to Live in Interesting Times : Canada's Changing Citizenship Regimes», Canadian Journal of Political Science, vol.30, no 4, 627-644.

JENSON, Jane (1989). «Paradigms and Political Discourse : Protective Legislation in France and the United States Before 1914», Canadian Journal of Political Science, vol. 22, no 2, 235-258.

JENSON, Jane (1986). "Gender and Reproduction: Or, Babies and the State», Studies in Political Economy, vol.20.9-46.

JENSON, Jane et Susan D. PHILLIPS (1996). «Regime Shift: New Citizenship Practices in Canada», International Journal of Canadian Studies, 14, 111-135.

LAMARCHE, Lucie (1998) $3^{e}$. "L'économie sociale: un modèle de développement au service de l'État désétatisé», Louise Boivin et Mark Fortier (dir) L'économie sociale: L'avenir d'une illusion, Montréal, Fides, 137-160.

LAMOUREUX, Diane (1998). «La panacée de l'économie sociale: un placebo pour les femmes?», Louise Boivin et Mark Fortier (dir) L'économie sociale : L'avenir d'une illusion, Montréal, Fides, 2553.

LAMOUREUX, Jocelyne (1994). Le partenariat à l'épreuve, Montréal, Éditions Saint-Martin.

LAVILLE, Jean-Louis (1995). «Économie solidaire, économie sociale et État social», Contre l'exclusion: repenser l'économie, Montréal, Presses de l'Université du Québec, 161-174.

LESEMANN, Frédéric et Pierre Joseph ULYSSE (1995). «Welfare, workfare et citoyenneté aux États-Unis», Lien social et Politiques - RIAC, vol. 34, 55- 62.

McALL, Christopher (1995). «Les murs de la citoyenneté: territoires d'exclusion et espaces de citoyenneté», Lien social et Politiques - RIAC, vol. 34, 81-92.

MICHAUD, Jacinthe (1997). «Le mouvement des centres de santé : grandeur et misère de la participation et stratégies politiques de transformation du discours de l'État», Nouvelles pratiques sociales, vol.10, no 1, 97-110.

MICHAUD, Jacinthe (1992). «The Welfare State and the Problem of Counter-Hegemonic Responses Within the Women's Movement», William K. Carroll (ed) Organizing Dissent : Contemporary Social Movement in Theory and in Practice, Toronto, Garamond Press, 200-214.

NOËL, Alain (1996). «Vers un nouvel État-providence? Enjeux démocratiques», Politique et sociétés, no 30, 3-27.

REGROUPEMENT PROVINCIAL DES MAISONS D'HÉBERGEMENT ET DE TRANSITION POUR FEMMES VICTIMES DE VIOLENCE CONJUGALE (1990). $A u$ grand jour, Madeleine Lacombe (rédaction), Montréal, Remue-ménage.

RELAIS-FEMMES (1985). Les rapports des groupes de femmes avec l'État. Compte rendu de la journée de réflexion organisée par Relais-femmes. 
ROCHE, Maurice (1995). «Rethinking Citizenship and Social Movements:Themes in Contemporary Sociology and Neoconservative Ideology», Louis Maheu (ed) Social Movements and Social Classes: The Future of Collective Action, London, Sage, 186-219.

SCOTT, Katherine (1996). «The Dilemma of Liberal Citizenship: Women and Social Assistance Reform in the 1990s», Studies in Policial Economy, no 50,7-36.

ST-HILAIRE (1996). "La production d'un sujet-femme adapté au développement: le cas de la recherche féministe aux Phillippines», Anthropologie et sociétés, vol. 20, no 1, 81-102.

TABLE FÉMINISTE FRANCOPHONE DE CONCERTATION PROVINCIALE DE L'ONTARIO (1998). Le travail obligatoire en Ontario: est-ce que cela vous inquiète?, Toronto, Forum sur le travail obligatoire, 30 mai.

WARING, Marlyn (1985). If Women Counted: A New Feminist Economics, San Francisco, Harper \& Row, Publisher.

WHITMORE, Elizabeth et Patrick KERANS (1988). «Partcipation, Empowerment and Welfare», Canadian Review of Social Policy, vol. 22, 51-60. 